\title{
Die Debatte über Priorisierung
}

und Rationierung in der

\section{Gesetzlichen Kranken-}

\section{versicherung: überfällig oder überflüssig?}

In der Gesetzlichen Krankenversicherung (GKV) gibt es keine legale oder legitime Rationierung, d. h. eine Vorenthaltung oder Verweigerung notwendiger medizinischer Behandlungsleistungen. Die aktuelle Rationierungs-Debatte fordert hier eine grundsätzliche Klarstellung heraus. Die Leistungen der GKV, auf die die Versicherten Anspruch haben, sind von der Notwendigkeit dieser Leistungen bestimmt, die ohne Rückgriff auf etwa akzeptable Kosten oder Ausgaben ermittelt wird. Die zur Bedarfsdeckung notwendigen Mittel werden unter Berücksichtigung von Wirtschaftlichkeitsreserven aufgebracht. Die Ausgaben der GKV steigen seit Jahren kontinuierlich an. Gleichwohl scheinen Unzufriedenheit und das Gefühl, in einem überreglementierten Mangelsystem zu arbeiten, bei vielen Leistungserbringern zuzunehmen. Als ein möglicher oder sogar der einzige Ausweg erscheint einigen Akteuren offensichtlich das Infragestellen bisher in Deutschland unstrittiger sozialstaatlicher Prinzipien. Doch was steckt hinter diesem Mut zum Tabubruch, weshalb soll die Gesundheitspolitik von der Notwendigkeit und Realität der Rationierung überzeugt werden? Um die GKV auf eine Basisversicherung zu schrumpfen? Um Vergütungserhöhungen durchzusetzen? Um von bestehenden Rationalisierungsreserven abzulenken? All das würde der GKV und unserem Gemeinwesen eher schaden als helfen. Die RationierungsDebatte sollte daher abgesagt und das kreative Potenzial und Engagement der Beteiligten in die Lösung der wirklichen Versorgungsprobleme investiert werden.

\section{Philipp Storz, Bernhard Egger}

Eine nicht ganz so neue ${ }^{1}$ "Rationierungs-Debatte“ wurde durch den viel beachteten Eröffnungsvortrag des Präsidenten der Bundesärztekammer, Prof. Dr. Hoppe, auf dem Ärztetag am 19. Mai 2009 in Mainz angefacht. Hoppe bemerkt ${ }^{2},[\ldots .$.$] wenn wir nicht mehr die ausreichenden Mittel$ für die Versorgung der Patienten bekommen, wenn also der jetzige Mangel von der Politik zementiert wird, dann müssen wir einfach offen und ehrlich darüber reden und zu gerechtem Verteilungsmechanismus kommen." Man wolle Ärzte, die „ [...] nur ihrem Gewissen und ihren Patienten verpflichtet sind, und nicht Allokationsjongleure in einem Rationierungssystem. " Offenbar sieht der höchste

Philipp Storz, Abteilung Medizin des GKV-Spitzenverbandes Bernhard Egger, Leiter der Abteilung Medizin des GKV-Spitzenverbandes
Repräsentant der Deutschen Ärzteschaft ein Mangelsystem als gegenwärtige Realität an und fordert mit dem Gestus des mutigen Tabu-Brechers, es müsse „offen und ehrlich“ über Leistungsbegrenzungen gesprochen werden. Dass hier „heimliche Rationierung“ am Werk gesehen wird, die von einer offenen „Priorisierung“ abgelöst werden müsse ist demgegenüber schon fast ein Detail, das aber ebenfalls eine kritische Würdigung verdient.

Es ist nicht nur diese eine Rede sowie eine inzwischen auf eine stattliche Zahl von Artikeln angewachsene Serie im Deutschen Ärzteblatt, mit der die Rationierungs-Debatte vorangetrieben wird. So wurden jüngst die Ergebnisse eines vom Bundesministerium für Bildung und Forschung (BMBF) finanzierten Projektes ${ }^{3}$ vorgestellt. Hier wird beansprucht, interdisziplinär kostensensible Leitlinien 


\section{THEMA}

entwickelt zu haben, um einer angenommenen Rationierungsnotwendigkeit zu entsprechen. Im Ergebnis wurde als eine mögliche Option vorgeschlagen, bestimmte Leistungen (im Bereich der kardiologischen Spitzenmedizin) für bestimmte Patientengruppen nicht mehr zur Verfügung zu stellen, weil das Verhältnis von Kosten und Nutzen bei diesen als zu ungünstig eingeschätzt wird ${ }^{4}$. Auch ein weiteres durch die Deutsche Forschungsgemeinschaft (DFG) finanziertes Projekt zur „Priorisierung in der Medizin“ behauptet in seiner Selbstdarstellung: „Es ist bereits heute absehbar, dass nicht mehr alle bisherigen Leistungen für alle gesetzlich Krankenversicherten finanziert werden können. Daraus ergibt sich die Notwendigkeit der Prioritätenbildung bzw. Auswahlentscheidung "5. Hier wird, wie in anderen Äußerungen zum Thema auch, die Rationierung apodiktisch für wahr, wirklich und unausweichlich erklärt.

Wir sehen die Gefahr, dass hier an einer selbsterfüllenden Prophezeiung gearbeitet wird. Unser Anliegen ist es aufzuzeigen, dass es eine legale oder legitime Rationierung medizinischer Leistungen in der heutigen Gesetzlichen Krankenversicherung (GKV) nicht gibt. Dazu ist es zunächst notwendig, eine präzise begriffliche Fassung dessen zu geben, was eine Rationierung medizinischer Leistungen darstellte. Dem kann dann der Leistungsanspruch der gesetzlich Krankenversicherten, wie er insbesondere im SGB V verbindlich bestimmt ist, gegenüber gestellt werden. Die vom Gesetzgeber getroffenen Regelungen zu Leistungsausschlüssen $\mathrm{z}$. B. von nicht verordnungspflichtigen Medikamenten oder die Regelungen zur Eigenbeteiligung beim Zahnersatz fassen wir dabei nicht als Rationierung in diesem Sinne auf. Der Gesetzgeber hat hierfür jeweils andere Begründungen im Sinne der Eigenverantwortung und Zumutbarkeit von Eigenbeteiligungen gegeben. Deren Höhe ist im internationalen Vergleich moderat, allerdings stellen sich auch in diesem Zusammenhang natürlich Fragen nach der Zumutbarkeit und deren Konsequenzen für den gleichmäßigen und gerechten Zugang zur Gesundheitsversorgung.

Wir versuchen ferner darzulegen, dass - bei allen Problemen den Bedarf und die Finanzierung adäquat zu regeln - auch in der Wirklichkeit der Leistungserbringung in der GKV keine systemische Rationierung angetroffen wird. Das bloße Vorenthalten medizinisch notwendiger Behandlungen durch einzelne Leistungserbringer oder Kostenträger, wenn und falls es vorkommt, stellt in diesem Sinne keine Rationierung in der GKV dar, sondern letztlich einen Willkürakt. Dieser ist ohne Rechtsgrundlage und vor derlei Willkür wurde bereits an prominenter Stelle jüngst gewarnt ${ }^{6}$.

\section{Der Begriff „Rationierung“ in der Medizin}

Der Aufsatz von Fuchs et al. ${ }^{7}$ definiert Rationierung in der Medizin als „Die Vorenthaltung medizinisch notwendiger Leistungen“. Das Gutachten zur „Über-, Unter- und Fehlversorgung“ des Sachverständigenrates aus dem Jahr 2001 definiert: „Rationierung kann verstanden werden als
Verweigerung oder Nichtbereitstellung von Behandlungsleistungen trotz Nachfrage und zugleich festgestelltem objektiven Bedarf." ${ }^{8}$ Beide Definitionen stimmen im Wesentlichen überein, da die (Behandlungs-) Notwendigkeit und der festgestellte, objektive Bedarf im Kern dasselbe bezeichnen. Im Kontext der Ausführung des Sachverständigenratsgutachtens 2001 wird dieser von einem bloß subjektiven Bedürfnis unterschieden. In der gesetzlichen Krankenversicherung ist die Behandlung nicht lediglich eine dem subjektiven Behandlungswunsch entsprechende „Dienstleistung am Kunden“, sondern es wird zunächst eine sinnvolle Untersuchung angestellt, um herauszufinden was denn tatsächlich medizinisch überhaupt angebracht ist. Zweifellos stehen das Behandlungsbedürfnis und ein Behandlungsbedarf in der ganz überwiegenden Zahl der Fälle nicht im Konflikt: Schließlich suchen die Patienten in der Regel medizinische Hilfe, weil sie Symptome beobachten oder Beschwerden haben. Die „medizinische Objektivierung “ besteht dann vor allem darin herauszufinden, welche Erkrankungsursache den Beschwerden zu Grunde liegt, ob und wie sie behandelbar ist. Eine Rationierung medizinischer Leistungen läge dann vor, wenn nach dieser Feststellung des Bedarfs eine Behandlung aus Kostengründen verweigert würde.

\section{Der legitime Behandlungsanspruch der Versicherten}

Der Behandlungsanspruch der Versicherten, wie er an verschiedenen Stellen im SGB V formuliert wird (wesentlich $\S \S 2,12,70$ SGB V) bezieht sich auf den medizinischen Bedarf. Es werden Qualität und Wirksamkeit der Leistungen nach dem anerkannten medizinischen Erkenntnisstand sowie eine Notwendigkeit der Leistungen, ein Nicht-Überschreiten des notwendigen Umfanges und eine angemessene Eigenverantwortung der Versicherten gefordert. Auch die Wirtschaftlichkeit wird als Kriterium genannt. In \$70 wird die Bedarfsgerechtigkeit und Gleichmäßigkeit der Versorgung sowie die Qualität und Humanität aufgeführt. Aus den Formulierungen wird deutlich, dass hier keine Einschränkung eines der Kriterien der angemessenen Leistungserbringung gegenüber einem anderen vorgesehen ist. Insbesondere das Wirtschaftlichkeitsgebot ist nach dem maßgeblichen Grundverständnis im Sozialrecht nicht etwa so konzipiert, dass Leistungen auf Grund der mit ihnen verbundenen Kosten bzw. Ausgaben ausgeschlossen sind. Vielmehr ist das Wirtschaftlichkeitsgebot so zu verstehen, dass Leistungen unwirtschaftlich sind, wenn sie den Patienten keinen Nutzen bringen oder demselben Bedarf zumindest nicht schlechter zu geringeren Kosten entsprochen werden kann.

Tatsächlich handelt es sich bei den genannten Begriffen bzw. Kriterien, die den Leistungsanspruch charakterisieren, um nicht im Einzelnen ihrem Inhalt nach bestimmte Begriffe, sodass jeweils Konkretisierungen erforderlich sind - was bei der Komplexität und Vielgestaltigkeit der modernen Medizin auch schwer anders denkbar ist. Diese Konkretisierungen werden in der überwiegenden Zahl der 
Fälle durch die Ärzte im Behandlungsalltag bestimmt ${ }^{9}$. Neben den professionellen Selbstverpflichtungen und Regeln sowie wissenschaftlichen Erkenntnissen bzw. Empfehlungen, wie sie z. B. in Leitlinien dargelegt sind, sind dabei die Richtlinien des Gemeinsamen Bundesausschusses (G-BA) zu beachten. Die Komplexität der wissenschaftlichen Entscheidungsgrundlagen und der nicht selten widerstreitenden Interessen bringt es mit sich, dass um Entscheidungen hier häufig intensiv gerungen wird. Es ist auch immer mit abweichenden Meinungen betreffend der Richtigkeit einzelner Entscheidungen zu rechnen. Die Prinzipien der Entscheidungsfindung, insbesondere auch die Forderung nach Evidenzbasierung, sind jedoch ein tragfähiger Konsens, ebenso wie die zulässigen Argumente und Diskussionsgrundlagen. Rationierungsargumente sind mit Sicherheit nicht darunter und sie haben auch keinen Platz in der Verfahrensordnung des Gemeinsamen Bundesausschusses. Dies gilt auch für die aktuell anhaltenden Diskussionen zur Kosten-Nutzen-Bewertung von Arzneimitteln. Entgegen mancher Behauptungen werden hier keine Leistungen rationiert, es werden lediglich entsprechend der gesetzlichen Grundlage nach §31 Abs. 2a SGB V ggf. Höchsterstattungsbeträge festgelegt - in der Erwartung, dass hiermit dem Nutzen und weiteren im Gesetz genannten Kriterien jeweils entsprechende angemessene Preise (ggf. auch als Basis von Verhandlungen des GKV-Spitzenverbandes mit Herstellern) ermittelt werden können.

\section{Die Forderung nach Wirtschaftlichkeit}

In Deutschland existiert kein Globalbudget, z. B. als festgeschriebener Anteil am Sozialprodukt mit der Folge, dass nur bezahlt wird, was dieses Budget hergibt. Die Budgets, die in einigen Versorgungsbereichen Teil der Vergütungssystematik sind, sind unter dem Gesichtspunkt dimensioniert, damit eine bedarfsentsprechende Versorgung realisieren zu können und erlauben Anpassungen. Die in §71 SGB V formulierte Forderung der Beitragssatzstabilität realisiert kein Globalbudget. Beitragserhöhungen, so wird in §71 Abs. 1 SGB V ausgeführt, sind nicht ausgeschlossen, falls die notwendige medizinische Versorgung auch nach Ausschöpfung von Wirtschaftlichkeitsreserven sonst nicht zu gewährleisten ist. Die Rationierung notwendiger medizinischer Leistungen ist hier also nicht gefordert und im Kontext der bereits genannten gesetzlichen Normen somit auch ausgeschlossen. Dass die Ausschöpfung von Wirtschaftlichkeitsreserven Vorrang vor der Erhöhung von Beiträgen hat, ist dabei unmittelbar einzusehen und wird im Rahmen der Rationierungs-Debatte auch gar nicht grundsätzlich angezweifelt.

\section{Die Verwirklichung einer bedarfsgerechten Versorgung}

An der rechtlich derart eindeutigen Situation liegt es sicher nicht, wenn trotzdem der Eindruck entsteht, es werde unter der Herrschaft eines Budgets Rationierung prakti- ziert. Man darf annehmen, dass hier mehrere Probleme zu Grunde liegen. Zum Einem besteht bei vielen Leistungserbringern eine erhebliche Handlungsunsicherheit auf Grund der Komplexität des Versorgungs- und Vergütungssystems. Abrechnungssystem, Honorarverteilungsmaßstäbe und Regelleistungsvolumen sind so kompliziert geworden, dass der einzelne Vertragsarzt die Übersicht zu verlieren droht. Viele einzelne Regelungen sind zu beachten, wenn er die konkreten und geeigneten medizinischen Leistungen zu Lasten der GKV auswählt. Der Arzneimittelmarkt und die Vermarktungsstrategien der Industrie sind undurchsichtig. Weiterhin sind Fragen der Angemessenheit von Vergütungen, Preisen oder Erstattungsbeträgen völlig anders als Fragen des medizinischen Bedarfs zu diskutieren. Schließlich stellt es eine erhebliche Herausforderung dar, den Bedarf sowie die erwartete Entwicklung dieses Bedarfs bevölkerungsbezogen zuverlässig zu ermitteln. Besonders die demografische Entwicklung sowie ein kaum präzise zu bestimmendes gestiegenes Anspruchsniveau der Versicherten werden als wesentliche Faktoren vermutet ${ }^{10}$. Es ist nach unserer Auffassung allerdings fahrlässig, diesen Entwicklungen einen Automatismus zu unterstellen und daraus düstere Prognosen einer Unfinanzierbarkeit unseres Gesundheitssystems abzuleiten. Unberücksichtigt bleiben bei solchen Prognosen häufig andere Einflussgrößen auf die Kostenentwicklung und erhebliche Unsicherheiten, die sich langfristig z. B. bezüglich der Frage der Expansion oder Kompression der Morbidität stellen: Können wir gesundes Altern verwirklichen oder werden wir vielmehr einem unabsehbaren Pflege- und Behandlungsbedarf gegenüberstehen? All diese Fragen verweisen zunächst nicht auf einen Bedarf an Rationierung sondern vielmehr auf eine weitere Erforschung sowie aktives Gegensteuern dort, wo es konkret Probleme gibt. Es sind in allen modernen Gesundheitssystemen wiederkehrende Fragen, deren Bearbeitung wir uns stellen müssen.

Auch in Organisations- und Strukturfragen bestehen vielfältige Herausforderungen, eine gute Versorgung zu organisieren und sicherzustellen. So steigt z. B. die Zahl der niedergelassenen Fachärzte seit vielen Jahren immer weiter $\mathrm{an}^{11}$, wobei deren räumliche Verteilung sich kaum als am Versorgungsbedarf orientiert erkennen lässt. Der bekannten Konzentration in attraktiven Ballungszentren steht zum Teil ein Mangel in relativ abgelegenen, ländlichen Räumen sowie in problematischen Bezirken von Ballungsräumen und Großstädten gegenüber. Die Zahl der Hausärzte stagniert dagegen seit Jahren. Bisherige Versuche, hier wesentliche Änderungen herbeizuführen, sind ohne Erfolg geblieben. Es sei jedoch an die Verpflichtung, eine gleichmäßige Versorgung zu verwirklichen, erinnert (§70 SGB V Abs. 1).

Die Erträge der niedergelassenen Ärzte (Praxisinhaber) nach Abzug der Praxiskosten liegen, was die GKVBehandlungen angeht, im Mittel bei gut 100.000 Euro pro Jahr; nimmt man die PKV und die weiteren Einnahmen dazu über 140.000 Euro pro Jahr, wobei insbesondere die Haus- und Kinderärzte deutlich geringere und einige hoch spezialisierte Facharztgruppen deutlich höhere Erträge verzeichnen $^{12}$. Alle berufstätigen Ärzte wollen ein nach 


\section{THEMA}

ihren Vorstellungen angemessenes Einkommen erzielen, was verständlich und legitim ist. Die hohe Arztdichte verweist aber zunächst eher auf Verteilungskämpfe als auf Rationierung. Die Frage der Verteilungsgerechtigkeit ärztlicher Einkommen hat sich in jedem Fall verschärft. Irritierend ist der Befund, dass die Zahl der Arztkontakte pro Versichertem und Jahr in Deutschland im internationalen Vergleich sehr hoch ist ${ }^{13}$. Mit Morbidität oder einer übergroßen Anspruchshaltung aller Deutschen alleine ist dies nicht zu erklären. Ärzte steuern über Wiedereinbestellungen und Versorgungsangebote den Bedarf der Patienten ganz wesentlich selbst mit.

Besonders bei Fachärzten gibt es außerdem einen zunehmenden Wandel im ärztlichen Selbstverständnis. Mit den „Individuellen Gesundheitsleistungen“ hat sich eine fragwürdige Praxis etabliert, die auch von Hoppe kritisch gesehen wird ${ }^{14}$. Selbst wenn nicht alle „IGeL“-Leistungen sinnlos und einige Arztgruppen auch nur in geringem Maße betroffen sind (wie z. B. gerade die Hausärzte), so gibt das Geschehen in diesem Markt doch Anlass zur Sorge. Die Versicherten werden auf breiter Front ${ }^{15}$ mit Angeboten konfrontiert, die meist durch das Fehlen eines Nutzens und durch ihre Unnötigkeit auffallen. Insbesondere zusätzliche Vorsorge- und Früherkennungsuntersuchungen werden angeboten ${ }^{16}$. Die Aussage, es handele sich um „nicht (mehr) von der Kasse bezahlte“ Leistungen ${ }^{17}$ signalisiert den Patienten, dass die Krankenkasse auf ihre Kosten spare. In diesem Vorgehen vieler Arztpraxen ist daher eine wesentliche Quelle der Sorge um vermeintlich grassierende Rationierung in der Bevölkerung auszumachen: Um den Verkauf unnützer Dinge zu befördern werden diese zu wertvollen Leistungen umetikettiert, die angeblich einer Rationierung seitens der Kassen anheim gefallen seien. Hier zeigt sich zudem, dass die Forderung nach Evidenzbasierung gerade nicht als eine Art heimlicher Rationierung dient. Sie verfolgt den Zweck, unnötige und ggf. schädliche Leistungen zu verhindern. Die Bewertung der gesundheitlichen Effekte und des Nutzens von Früherkennungsprogrammen ist anspruchsvoll und die differenzierte Diskussion, z. B. des mit sehr hohen Qualitätsstandards eingeführte Mammographie-Screenings, zeigt dies auch. Die Vorstellung, mehr „nachschauen und checken“ sei immer besser, ist irrig ${ }^{18}$.

Mindestens ebenso problematisch sind jedoch Leistungen, die im Rahmen der GKV erbracht werden und deren Nutzen nicht erwiesen ist. So ist z. B. die Arthroskopie am Kniegelenk bei Arthrose-Patienten ein häufiger Eingriff, obwohl wissenschaftlich belastbar gezeigt wurde, dass diese für den Patienten nichts Positives erbringt ${ }^{19}$. Auch z. B. bei der Diagnose und Behandlung der sehr weit verbreiteten Rückenschmerz-Leiden besteht sowohl der dringende Verdacht, dass unnötig viele bildgebende Untersuchungen durchgeführt werden - trotz expliziter Warnungen in Leitlinien $^{20}$ - als auch große Unsicherheit über den Wert einer Vielzahl angebotener Behandlungsmethoden ${ }^{21}$. Diese Liste lässt sich zweifellos verlängern. Zudem ist nach wie vor davon auszugehen, dass viele therapeutische und diagnostische Technologien noch gar nicht auf ihre Nützlichkeit bzw. auf ihr Nutzen-Schadens-Verhältnis hin untersucht wurden und hier Verbesserungspotenziale schlummern ${ }^{22}$. Methoden, die medizinische Fortschritte realisieren können, werden häufig außerhalb ihres eigentlichen Indikationsgebietes angewandt. Dies könnte z. B. für invasive kardiologische Eingriffe gelten, insbesondere die perkutanen Koronarinterventionen, ggf. mit Stent-Implantation. Auch von diesen wird vermutet, dass sie in Deutschland zu häufig eingesetzt werden. Die Vermutung wird durch einen internationalen Vergleich gestützt: Während in Deutschland für das Jahr 2007 rund 550 stationäre Eingriffe pro 100.000 Personen verzeichnet wurden, erreichte diese Rate kein anderer europäischer OECD-Mitgliedsstaat auch nur annähernd (im Mittel wurden hier kaum mehr als 200 Prozeduren pro 100.00 Einwohner gezählt $)^{23}$ - ohne, dass die alters- und geschlechtsstandardisierte Mortalitätsrate ischämischer Herzkrankheiten in Deutschland in Folge dieser intensiveren Therapie auffällig gering wäre ${ }^{24}$. Umso bemerkenswerter ist deshalb, dass gerade eine Variante dieser Art von Interventionen (die Implantation eines medikamentenbeschichteten Koronarstents) ein exemplarischer Gegenstand der erwähnten Versuche, „Kostensensible Leitlinien“ zu entwickeln gewesen ist.

Diese und weitere mögliche Beispiele, die unterschiedliche Leistungssektoren betreffen können, zeigen eine generelles Problem an. Wie sicher können wir uns sein, dass die Leistungen, wie sie gegenwärtig in der Versorgung erbracht werden, wirklich dem Bedarf entsprechen? - Sowohl in wichtigen Bereichen der Arzneimittelversorgung als auch bei den Krankenhausleistungen gilt heute zunächst einmal das Prinzip, dass die geforderten Preise bezahlt und die durchgeführten Leistungen erstattet werden. Durch die Defizite bzw. das „Hinterherlaufen“ insbesondere auch der Nutzenbewertung sind hier die Risiken für die Patienten als auch betreffend der Finanzierung erheblich.

Sollten wir uns in dieser Situation nicht endlich einmal ernsthaft und gemeinsam darum bemühen, die Nutzenfrage zu prüfen und entsprechende Konsequenzen für die Versorgungsrealität zu ziehen?

\section{Angemessene Preise und Vergütungen}

Betrachtet man Vergütungen, Erstattungsbeträge und Preise, ist neben empirischen Unsicherheiten auch das normative Gerüst und der volkswirtschaftliche Gesamtzusammenhang von offenkundiger Bedeutung. Wie viel verdient ein Arzt zu verdienen? Welches sind angemessene Vergütungen für Krankenhausleistungen, Arzneimittel, Medizintechnik? Viele Bereiche der Finanzierung und Vergütungen wurden in den vergangenen Jahren kontinuierlich umstrukturiert. Auch wenn man kurzfristig bisweilen an diesen „Dauerbaustellen“ verzweifeln mag: In längerfristiger Perspektive sind die Entwicklungen in einigen Bereichen durchaus bemerkenswert. So sind sowohl im Hinblick auf den Kassenwettbewerb (mit dem morbiditätsorientierten Risikostrukturausgleich) als auch auf dem Gebiet der Vergütung (in der stationären Versorgung mit dem diagnosebezogenen Fallpauschalensystem und in der 
vertragsärztlichen Versorgung dem Einstieg in die Morbiditätsorientierung) wesentliche Reformschritte gegangen worden. Diesen ist gemeinsam, dass sie Finanzierungsund Vergütungsströme nach transparenten Kriterien neu ordnen, die sowohl Bedarfskomponenten (Morbiditätsorientierung) als auch rationale Vergütungssystematiken beinhalten. Weitere Elemente, z. B. zur Realisierung einer qualitäts- und leistungsorientierten Vergütung, werden gegenwärtig diskutiert.

Vergleichbar rationale Instrumente fehlen in anderen Versorgungsbereichen, z.B. betreffend die nicht festbetragsfähigen Arzneimittel, insbesondere bezüglich extrem teurer Präparate etwa zur Behandlung von Krebserkrankungen oder Erkrankungen des Immunsystems. Hier warnte der Vorsitzende der Arzneimittelkommission der Deutschen Ärzteschaft, Prof. Ludwig, mehrfach vor einer drohenden „Unbezahlbarkeit“25, sollte sich die Entwicklung der jüngeren Vergangenheit im Hinblick auf die Preisgestaltung zukünftig fortsetzen ${ }^{26}$. Unverdächtig, die möglichen Sorgen einzelner Finanzierungsträger zur Grundlage zu machen, wird hier tatsächlich über die Nutzenfrage hinaus die Preisfrage gestellt, die in der RationierungsDebatte in der Regel nicht gestellt wird ${ }^{27}$ : Was könnte z. B. der angemessene Preis eines Arzneimittels mit bestimmten Charakteristika sein? Man könnte, um eine Verengung des ethisch geschulten Blicks zu vermeiden, ja auch die die (Gegen-)Frage stellen: Wie ist es ethisch zu bewerten, wenn ein exorbitanter („unbezahlbarer“) Preis gefordert wird?

\section{Zur fragwürdigen Relevanz der "Rationierung durch Priorisierung"}

Einige der Beiträge zur aktuellen Rationierungs-Debatte unterscheiden zwischen Rationierung und Priorisierung, ohne dass das jeweils Gemeinte und die Beziehung dieser Begrifflichkeiten zueinander immer ganz klar ist. Einige scheinen Priorisierung statt Rationierung zu fordern. Dieser Gedanke liegt dem Anschein nach auch der bereits erwähnten Rede von Hoppe auf dem Ärztetag 2009 zu Grunde. Eine klare Unterscheidung zu treffen bemüht sich der schon zitierte Artikel von Fuchs et al. aus dem Deutschen Ärzteblatt „Rationalisierung, Rationierung und Priorisierung - was ist gemeint?“. In diesem Artikel wird ausgeführt, Priorisierung bestehe in der Aufstellung einer Rangfolge: „Dabei entsteht eine mehrstufige Rangreihe, in der nicht nur Methoden, sondern auch Krankheitsfälle, Kranken- und Krankheitsgruppen, Versorgungsziele und vor allem Indikationen (das heißt Verknüpfungen bestimmter gesundheitlicher Probleme mit zu ihrer Lösung geeigneten Leistungen) in einer Rangfolge angeordnet werden können. "Entscheidend ist, wie die Beziehung der Priorisierung zur Rationierung aufgefasst wird. Hier konzipiert der Artikel eine klare Abhängigkeitsbeziehung: Priorisierung dient als ein Instrument der Rationierung. Wenig prioritäre Leistungen könnten im Sinne wirksamer Rationierung dann entfallen. Diese Abhängigkeitsbeziehung, in der die Priorisierung instrumentellen Charakter hat, ist auch durchaus plausibel - so man denn Rationie- rung für unvermeidlich hält. Im Übrigen wird in Bezug auf die Zuweisung unterer Rangplätze als Ergebnis einer Priorisierung ausgeführt: „Theoretisch sollen sich am Ende dieser Rangreihe solche Verfahren wiederfinden, die keine nachweisbare Wirkung haben. "Diese wenig geglückte Formulierung - weshalb sollten wirkungslose Interventionen überhaupt in Betracht gezogen werden? - zeigt noch einmal die wesentliche Problematik der Diskussionen. Nur wenn eine Rationierung bereits als gegeben bzw. unmittelbar notwendig unterstellt wird, könnte eine Priorisierung möglicherweise hilfreich sein. Priorisiert man gleichsam auf „Vorrat“ so hat dies nur Sinn, wenn man eine Rationierung letztlich befürwortet. In der gegenwärtigen Diskussion droht das grundsätzlich plausible, hypothetische Verhältnis von Rationalisierung und Priorisierung dagegen auf den Kopf gestellt zu werden. Auch das oben skizzierte Projekt zur Entwicklung sogenannter „Kostensensibler Leitlinien“ krankt an dieser Verkehrung. Wird nicht rationiert, so bedarf es auch keiner Priorisierung im geschilderten Sinne, da auch weniger prioritäre Leistungen zur Verfügung stehen - wenn sie denn notwendig sind - was die Annahme und schließlich auch die Überprüfung einer nachweisbaren Wirkung voraussetzt.

Von solchen Überlegungen nicht betroffen sind offenbar alltäglich notwendige Entscheidungen über Prioritäten. Nicht immer kann alles gleichzeitig gemacht werden. Wenn ein Arzt z. B. diejenigen Patienten aus seinem Wartezimmer zuerst aufruft, die akute Beschwerden haben, gegenüber anderen, die lediglich eine Routineuntersuchung benötigen, so stellt dies technisch gesehen sicherlich eine Priorisierung der Patienten mit akuten Beschwerden dar. Niemand wird jedoch, so ist zumindest zu hoffen, deshalb eine Rationierungs- oder Priorisierungs-Debatte führen wollen.

Einen anderen Akzent setzt Schmacke in seinem Aufsatz „Rationierung im Gesundheitswesen. Hartnäckige Routinen verschwenden Ressourcen “28. Er verweist darin auf die faktische „Posteriorisierungspraxis“ eines Gesundheitssystems, das z. B. „eine nahezu endlose Abfolge von Chemotherapie-Serien " durchführt, gleichzeitig aber „Kernbereiche der Palliativmedizin wie eine angemessene Schmerztherapie" vernachlässigt. Diese Liste ließe sich zweifellos noch um weitere Beispiele verlängern. Hierbei kann die „kurative Maximaltherapie“ im Blickpunkt stehen. Eben so kann es sich jedoch auch um den unbefragten, „routinemäßigen“ Einsatz technologielastiger Intervention bei auch konservativ gut behandelbaren, teilweise selbstlimitierenden oder spontan remittierenden Gesundheitsproblemen handelt. Hier wird ein Kernproblem unseres Gesundheitssystems angesprochen. Wir sehen dies allerdings sinnvoller unter dem Begriff Fehlversorgung erfasst. Es handelt sich dabei ja gerade nicht um eine Vorenthaltung oder Posteriorisierung von Leistungen aus Kostengründen sondern um Fragen der grundlegenden Orientierung ärztlichen Handelns an bestimmten Therapiezielen und Leitbildern. Diese Überlegungen verweisen unseres Erachtens zu Recht darauf, dass die Fragen des Bedarfs im Kontext des möglichen, notwendigen und 


\section{THEMA}

wünschbaren in der Medizin fortlaufend diskutiert und erwogen werden müssen.

\section{Die Fragwürdigkeit einer "heimlichen Rationierung"}

Wie wichtig die Klärung des Rationierungsbegriffs ist zeigt auch ein weiterer, besonders heimtückischer Aspekt der Rationierungs-Debatte: die Unterstellung, eine verdeckte Rationierung sei bereits heute fester Bestandteil des Versorgungsalltags. Dies wird durch die schon genannten Äußerungen der Ärzteschaft in deren „Ulmer Papier“ von 2008 bekräftigt, wenn ausgeführt wird: „Der Zielkonflikt zwischen ärztlicher Sorgfaltspflicht und Wirtschaftlichkeitsdruck belastet das Patient-Arzt-Verhältnis inzwischen in einem unerträglichen Ausmaß“. Das Argument lautet hier und in ähnlichen Äußerungen ganz einfach: Rationierung bei einzelnen Behandlungsentscheidungen sei unvermeidlich, da die Finanzmittel (der GKV) eben nicht ausreichten. Die Heimlichkeit wird dann in der Regel sowohl darin gesehen, dass diese Rationierungsnotwendigkeit politisch verschwiegen werde und im Vertrauensverhältnis zwischen Arzt und Patient ebenso allenfalls heimlich stattfindet.

Doch wie steht es mit empirischen Belegen zur verdeckten Rationierung? Es gab durchaus in der Vergangenheit Versuche, die Frage einer verdeckten Rationierung zu beantworten $^{29}$ - auf der Grundlage von Befragungen von Ärzten, mit dem wiederkehrenden Ergebnis jeweils hoher Anteile von Befragten, die angeben, sie seien zur Rationierung gezwungen. Dabei war allerdings auch hier nicht in allen Fällen erkennbar, was die Befragten unter Rationierung eigentlich verstehen. Zudem wird in den Befragungen schon im Ansatz nicht hinreichend klar, bei welchen Patienten und Indikationen welche Leistungen tatsächlich vorenthalten worden sein sollen ${ }^{30}$. Auch die jüngst publizierten Ergebnisse einer Befragung ${ }^{31}$, die in der breiteren Öffentlichkeit ein Echo gefunden hat, beanspruchen eine weit verbreitete Rationierung am Krankenbett aufgedeckt zu haben. Die gut fünfhundert befragten Ärzte aus den Bereichen Kardiologie und Intensivmedizin gaben zu 13\% an, häufig (mindestens einmal pro Woche) rationieren ${ }^{32} \mathrm{zu}$ müssen, selten (einmal pro Monat oder seltener) zu 64\% und nie zu 23\%. Zwar wurde in dieser Befragung einerseits eine adäquate Umschreibung bzw. Begriffsdefinition der Rationierung in dem auch von uns zugrunde gelegten Sinne verwendet. Es wird jedoch andererseits eingeräumt, dass es sich dabei um subjektive Einschätzungen der befragten Ärzte handele, da aus „Praktikabilitätsgründen“ weder konkrete Beispiele noch die Evidenz für die Nützlichkeit der vorenthaltenen Maßnahme abgefragt wurden.

Diese Sachlage verweist auf ein Kernproblem: in der Mehrzahl der Fälle müssen einzelne Ärzte ad hoc bestimmen, was die Behandlung des jeweiligen Patienten erfordert. Das ist einerseits eine sehr große fachliche Herausforderung. Bedenkt man hier jedoch andererseits auch die empörten Reaktionen, die z. B. Nutzenbewertungen medizinischer Interventionen durch das IQWiG regelhaft hervorrufen, so ist die Frage naheliegend, ob in vielen Einzelfällen die Meinungen über nicht adäquat zu erbringende Leistungen auch auf einer verzerrten Auffassung über deren tatsächliche Nützlichkeit und Notwendigkeit beruhen können. Damit soll weder gesagt werden, dass sich die Ärzte nicht unter beträchtlichen Anstrengungen bemühen, ihre Patienten gut und umfassend zu versorgen, noch dass es in der Versorgungsrealität Fehl- und auch Unterversorgung gibt. Dann sind aber weitere Anstrengungen insbesondere der Versorgungsforschung notwendig. So müsste insbesondere die Frage gestellt werden, welche Leistungen konkret nicht erbracht werden konnten und auf welche Gründe dies zurückzuführen war. Mit einer allgemeinen Aussage kann es bei einem solch gravierenden Problem doch nicht sein Bewenden haben. Warum erlahmt an dieser Stelle das Interesse der Forscher?

\section{Wozu die Rationierungs-Debatte geführt wird}

Zwar ist die Frage „Wem nützt die Rationierungs-Debatte"? durchaus naheliegend. Es soll hier jedoch nicht behauptet werden, sie würde um solchen möglichen Nutzens willen geführt, auch wenn dieser Eindruck insbesondere bei vielen Vertretern der pharmazeutischen Industrie sehr nahe liegt, die die evidenzbasierte Medizin und den Gemeinsamen Bundesausschuss als mehr oder weniger verdeckt arbeitende Rationierungsinstrumente begreifen ${ }^{33}$. Auch in Zusammenhang mit der plakativen Vermarktung von IGeL-Leistungen erscheint die Stoßrichtung ziemlich klar. Doch auch außerhalb dieser auf den ersten Blick durchsichtigen Verwertungszusammenhänge erscheint uns die Frage nach Risiken und möglichen Konsequenzen der gesamten Debatte bedeutsam. Ihre Folgen sind auch in der Öffentlichkeit spürbar. Jüngste Zeitungsartikel dokumentieren ein erhebliches Ausmaß an Verwirrung und Ratlosigkeit. ${ }^{34}$ Authentische Erlebnisse mit schlechter Versorgung mischen sich mit Ängsten, es liege einfach alles an fehlendem Geld.

Käme es soweit, dass wesentliche Entscheidungsträger der Gesundheitspolitik an die Wirklichkeit der Rationierung glaubten, so ergäben sich grundsätzlich zwei Handlungsmöglichkeiten: Erstens wäre es denkbar, die gegenwärtigen Vergütungen und Erstattungsregeln so zu verändern, dass in der Wahrnehmung derjenigen, die Rationierung beklagen, diese nicht mehr notwendig wäre. Wer am lautesten die Rationierung beklagt, könnte sich als erster Nutznießer aus solchen Veränderungen betrachten. Von einer effizienteren Versorgung würde man sich so allerdings mehr denn je entfernen. Fragen der Bedarfsgerechtigkeit träten völlig in den Hintergrund. Zweitens wäre denkbar, mit Hilfe erheblicher rechtlicher Eingriffe in das Regelwerk der GKV Rationierung als Mittel der Gesundheitspolitik hoffähig zu machen - sofern man verfassungsrechtlich am Ende nicht doch damit scheitert. Auf diese Weise könnte die GKV auf die Erbringung von „Basisleistungen“ reduziert oder Festbetragsregelungen könnten auf zahlreiche Leistungsbereiche ausgedehnt werden. Für 
die dann notwendigen Zusatzleistungen würden zweite Gesundheits- und Zusatzversicherungsmärkte eröffnet. $\mathrm{Ob}$ und wie Solidarität und soziale Gerechtigkeit besonders auch beim Zugang zum medizinischen Fortschritt - der ja gegenwärtig vom Leistungsanspruch der Versicherten umfasst ist - gewährleistet werden könnte stünde dann allerdings zur Disposition.

\section{Fazit}

Die von Hoppe geforderte offene und ehrliche Diskussion über die Ressourcenprobleme in unserem Gesundheitssystem sollte in der Tat geführt werden - allerdings auch unter Berücksichtigung unangenehmer, in der Ärzteschaft bisher gerne aus dem Blick verlorener Fakten. Hierzu gehören in erster Linie die genannten Probleme inädquater Versorgungsstrukturen und ärztlicher Verhaltensmuster zur Steuerung der Inanspruchnahme, sowie innerärztliche Verteilungskämpfe. Hier fallen Ressourcenprobleme nicht vom Himmel sondern sie sind, zumindest teilweise, auch hausgemacht. Es ist zu konstatieren, dass es der gemeinsamen Selbstverwaltung von Ärzten und Krankenkassen bisher nicht gelungen ist, eine wirklich am Bedarf orientierte Niederlassungssteuerung in der vertragsärztlichen Versorgung und einen tragfähigen Interessensausgleich zwischen Haus- und Fachärzten herbeizuführen. Die RationierungsDebatte ist offenkundig nicht nur kein geeignetes Mittel diese Probleme anzugehen, sie könnte sogar dazu beitragen, angemessene Lösungsversuche zu behindern

Weitere strukturelle Probleme kommen hinzu. So ist es eine ernüchternde Beobachtung, dass es den für die Fragen der Weiterbildung zuständigen Ärztekammern nicht gelungen ist, die Allgemeinmedizin als für den Nachwuchs attraktives Fach zu profilieren und in der gesamten Ärzteschaft die Orientierung an evidenzbasierten Nutzenabwägungen als genuinen Bestandteil ärztlicher Tätigkeit zu verankern. Warum setzt sich die Ärzteschaft nicht vehementer für evidenzbasierte Nutzenbewertungen oder z. B. Arzneimittel-Positivlisten ein und setzt stattdessen auf Rationierung?

Die Kernfrage vor dem Hintergrund dieser Fülle von Herausforderungen ist letztlich, in welche Richtung wir die kreativen Potentiale der Wissenschaft und das Engagement der in der Selbstverwaltung Handelnden richten wollen. Die internationalen Erfahrungen mit Rationierung und Priorisierungslisten sind sowohl was deren Erstellung als auch deren Anwendung in der Versorgungsrealität angeht wenig überzeugend. Eine Lösung für die Strukturprobleme im deutschen Gesundheitswesen und der von vielen erhoffte Befreiungsschlag aus den Restriktionen ärztlicher Tätigkeit sind sie sicher nicht. Am Ende wird politisch zu entscheiden sein, ob der Weg in die Rationierung oder in Richtung von Strukturreformen im Gesundheitswesen beschritten wird.

Im Grundsatz hat sich auch der neue Gesundheitsminister Rösler für die Beibehaltung eines umfassenden solidarischen Leistungskataloges ausgesprochen ${ }^{35}$. Das Postulat der Orientierung der Ärzteschaft am Wohl des Patienten und nicht am Mammon wurde auch von Hoppe aktuell wieder betont ${ }^{36}$. Handlungs- und Reformbedarf besteht nach unserer Auffassung nicht nur in Finanzierungsfragen unseres sozialen Krankenversicherungssystems. Die Frage nach dem Nutzen medizinischer Interventionen sollte im gesamten Bereich der Medizin sehr viel stärker in den Vordergrund gestellt werden. Das ist mühsam und wird zu erheblichen Konflikten führen, da die Interessen einflussreicher Gruppen tangiert werden. Nach unserer Überzeugung ist dieser Prozess unverzichtbar.

\section{Fußnoten:}

1 „Priorisierung medizinischer Leistungen im System der Gesetzlichen Krankenversicherung (GKV)“, Kurzfassung der Stellungnahme der Zentralen Ethikkommission bei der Bundesärztekammer; Deutsches Ärzteblatt, Jg. 104, Heft 40, 5. Oktober 2007, A2750 ff. sowie: „Gesundheitspolitische Leitsätze der Ärzteschaft - Ulmer Papier - Beschluss des 111. Deutschen Ärztetages 2008 (www.bundesaerztekammer.de [Zugriff 01.12.2009])

2 Die Zitate basieren auf dem von der Bundesärztekammer publizierten Redemanuskript „Rede des Präsidenten der Bundesärztekammer und des Deutschen Ärztetages, Prof. Dr. Jörg-Dietrich Hoppe, zur Eröffnung des 112. Deutschen Ärztetages: „Verteilungsgerechtigkeit durch Priorisierung - Patientenwohl in Zeiten der Mangelverwaltung“. Diese erfolgt unter dem Hinweis „Es gilt das gesprochene Wort“ (http://www. bundesaerztekammer.de [Zugriff 01.12.2009])

3 „BMBF-Forschungsverbund „Allokation“”(http://www.iegm.unituebingen.de [Zugriff am 02.12.2009])

4 „Die Arbeit des interdisziplinären Forschungsverbunds „Allokation“ 2006-2009 im Überblick“. Präsentation von Georg Marckmann am 30.10.2009 (http://www.gerechte-gesundheit.de/uploads/tx_igknb/ lecture/Praesentation_Marckmann-KSLL_301009.pdf [Zugriff am 02.12.2009])

5 http://www.priorisierung-in-der-medizin.de [Zugriff am 01.12.2009]

6 Dannecker et al. 2009 (Deutsches Ärzteblatt, Jg. 106 , Heft 41, 9. Oktober 2009, A2007 ff.)

7 Fuchs et al. 2009 (Deutsches Ärzteblatt, Jg. 106, Heft 12, 20. März 2009, A554 ff.)

8 SVR 2001, Bd. III, Absatz 23

9 SVR 2001, Bd. III, Absatz 25

10 dem Faktor der „medizinischen Innovationen“, der zweifellos von Bedeutung ist, wird über Bewertungen zu Nutzen und Notwendigkeit im G-BA, wie oben skizziert, Rechnung getragen

11 Dabei hat seit Anfang der neunziger Jahren (1990) bei im wesentlichen unveränderter Bevölkerungszahl die Zahl der niedergelassenen Ärzte in Deutschland um 50 Prozent zugenommen.Die Zahl der berufstätigen Ärzte hat insgesamt um 34 Prozent zugenommen (Quelle: Kassenärztliche Bundesvereinigung 2009, GKV-SV eigene Berechnungen)

12 Kostenstruktur bei Arzt- und Zahnarztpraxen; Kostenstrukturerhebung 2007, Fachserie 2 Reihe 1.6.1, Statistisches Bundesamt. In Bezug auf die Erträge aus der GKV wurde hier davon ausgegangen, dass die Kosten der Praxis in gleichem Maße zur Erzielung der GKV-Einnahmen wie zur Erzielungen weiterer Einnahmen anfallen.

13 „GEK-Report. Deutsche gehen im Schnitt 18-mal im Jahr zu Arzt“ (Deutsches Ärzteblatt, Jg. 106 , Heft 4, 23. Januar 2009, A108); Grobe, T.G., Dörning, H. und Schwartz, F.W. (2008): GEK-Report ambulantärztliche Versorgung 2008 (Gmünder ErsatzKasse), St. Augustin.

14 „,Ärzte sind keine Kaufleute, und sie verkaufen keine Ware“, Interview mit Prof. Hoppe (Deutsches Ärzteblatt, Jg. 105, Heft 3, 18. Januar 2008, A67 ff); , ,Igeln“ bringt Ärzten mehr als eine Milliarde“, Samir Rabbata (Deutsches Ärzteblatt, Jg. 105, Heft 36, 5. September 2008)

15 26,7\% der vom Wissenschaftlichen Institut der AOK(WidO) im Jahr 2008 befragten 3.000 Versicherten wurden solche Leistungen angeboten. In rund zwei Dritteln der Fälle ging die Initative vom Arzt aus (WidO-Pressemitteilung vom 19. August 2008, www.wido.de Zugriff: 15.12.2009)

16 Anhang zur WidO-Pressemitteilung vom 19. August 2008, www.wido.de Zugriff: 15.12.2009 
17 Zok K, Schuldinski W. Private Zusatzleistungen in der Arztpraxis. Ergebnisse aus Patientenbefragungen. Wissenschaftliches Institut der AOK (WIdO), Bonn, Oktober 2005.

18 IQWiG (2009) „Was Sie über Früherkennungsuntersuchungen wissen sollten“ (http://www.gesundheitsinformation.de, Zugriff 15.12.2009.

19 Laupattarakasem W, Laopaiboon M, Laupattarakasem P, Sumananont C. Arthroscopic debri-dement for knee osteoarthritis. Cochrane Database of Systematic Reviews 2008, Issue 1. Art. No.: CD005118. DOI: 10.1002/14651858.CD005118.pub2.); Kirkley, A. et al. (2008) „A Randomized Trial of Arthroscopic Surgery for Osteoarthritis of the Knee“, New England Journal of Medicine 359;11;1097-1107; Mosley, J. B. et al. (2002) „A CONTROLLED TRIAL OF ARTHROSCOPIC SURGERY FOR OSTEOARTHRITIS OF THE KNEE“. New England Journal of Medicine 347;2;81-88

20 Deutsche Gesellschaft für Allgemeinmedizin (DEGAM) „DEGAM Leitlinie Kreuzschmerzen (gekürzte Internetfassung)“, 2003 (www.degam. de/leitlinien; Zugriff 15.12.2009). Eine „Nationale Versorgungsleitlinie“ befindet sich in der Bearbeitung und wird voraussichtlich zu Beginn des Jahres 2010, zunächst in einer Konsultationsfassung, veröffentlicht.

21 American Pain Society \& The American Academy Of Pain, 2008. Guideline for the Evaluation and Management of Low Back Pain. Evidence Review. , 1-482.; Ibrahim, T., Tleyjeh, I. M., \& Gabbar, O. (2008). Surgical versus non-surgical treatment of chronic low back pain: a meta-analysis of randomised trials. International orthopaedics, 32(1), 107-13. doi: 10.1007/s00264-006-0269-6.; Staal JB, de Bie R, de Vet HCW, Hildebrand J, Nelemans P. Injection therapy for subacute and chronic low-back pain. Cochrane Database of Systematic Reviews 2008, Issue 3. Art. No.: CD001824. DOI: 10.1002/14651858.CD001824.pub3.; Keller, A., Hayden, J., Bombardier, C., \& van Tulder, M. (2007). Effect sizes of non-surgical treatments of non-specific low-back pain. European spine journal: official publication of the European Spine Society, the European Spinal Deformity Society, and the European Section of the Cervical Spine Research Society, 16(11), 1776-88. doi: 10.1007/s00586-007-0379-x.

22 Joshi NP, Stahnisch FW, Noseworthy TW. Reassessment of Health Technologies: Obsolescence and Waste. Ottawa: Canadian Agency for Drugs and Technologies in Health; 2009.; Franklin GM, Budenholzer BR. (2009) Implementing Evidence-Based Health Policy in Washington State. New England Journal of Medicine, 361;18; 1722-1725

23 OECD-Health Data 2009 („OECDHealthData_FrequentlyRequestedData.xls"; (www.irdes.fr;Zugriff 21.12.2009)

24 HFA (Health for All)-Database der Weltgesundheitsorganisation (WHO), Bezugsjahre 2005/2006 (http://data.euro.who.int/hfadb/, Zugriff: Juli 2009]

25 „Milliardengeschäft Krebsmedikamente. Wie die Pharmaindustrie mit Scheininnovationen Kasse macht“ (Report Mainz, www.swr.de, Zugriff 16.12.200); „Ärzten fehlen neutrale Arznei-Informationen“ (Interview mit Prof. Ludwig, Gesundheit und Gesellschaft, Ausgabe 6/07, 10. Jahrgang);

26 Bach, P. E. (2009) „Limits on Medicare's Ability to Control Rising Spending on Cancer Drugs“, New England Journal of Medicine 360;6;626633

27 Diese Frage ist erstaunlicherweise offenbar nicht selbstverständlich und Diskussionen unterstellen häufig mehr oder weniger, dass dieser Preis gleichsam „naturwüchsig“ gegeben sei . Das dies keineswegs der Fall ist, zeigt im internationalen Kontext nicht zuletzt eine selbstkritische Arbeit zum Umgang des NICE mit Innovationen in der die „Preisfrage“ als „[...] factor which is forever in the background“ (Sir Ian Kennedy „APPRAISING THE VALUE OF INNOVATION AND OTHER BENEFITS. A SHORT STUDY FOR NICE“, 22. Juli 2009, Seite 15) charakterisiert wird.

28 Schmacke, N. G+G Wissenschaft, Ausgabe 1, 2010, S.7

29 Kern et al. 1999 (Deutsches Ärzteblatt, Jg. 96 , Heft 3, 22. Januar 1999, A113 ff.);

Boldt \& Schölhorn 2008 (Deutsches Ärzteblatt, Jg. 105 , Heft 19, 9. Mai 2008, A995 ff.)

30 Am detailreichsten ist hier noch die, allerdings 10 Jahre alte, Befragung von Lesern des Deutschen Ärzteblatts von Kern et al. 1999. Hier wird die Hitliste („Diagnoseverfahren, die aus Sicht niedergelassener Ärzte nicht mehr abgerechnet werden können“) von Sonographien, Laborleistungen, EKG und Röntgen angeführt bzw. von Heilmitteln, Patientengesprächen und Arzneimittelverordnungen („Therapeutische Leistungen, die aus Sicht niedergelassener Ärzte nicht mehr abgerechnet werden können“). In dieser Studie war es allerdings der Interpretation der Befragten überlassen, was Rationierung sei.

31 Strech D, Danis M, Löb M, Marckmann G. Ausmaß und Auswirkungen von Rationierung in deutschen Krankenhäusern. Ärztliche Einschätzungen aus einer repräsentativen Umfrage. Dtsch Med Wochenschr 2009; 134: 1261-1266.

32 Es wurde nicht explizit nach „Rationierung“ gefragt, sondern - in Übereinstimmung mit dem Begriff der Rationierung, der auch im vorliegenden Beitrag zugrunde gelegt wurde - „Wie häufig haben Sie in den letzten 6 Monaten eine für den Patienten nützliche Maßnahme aus Kostengründen nicht durchgeführt bzw. durch eine preiswertere zu zugleich weniger effektive Maßnahme ersetzt?“

33 Sickmüller B, Lietz C: Konkretisierung des Leistungskatalogs der GKV. Gesellschaftspolitische Kommentare Sonderausgabe Nr. 2/09, November 2009, Seite 61f.

34 „DIE ZEIT“ vom 10.12.2009 („Medizin am Limit“, „Feilschen ums Rezept“); „Focus“ vom 14.12.2009 („Was Gesundheit kosten darf“)

35 Interview mit der Zeitung „Der Tagesspiegel“ (20.12.2009)

36 Hoppe JD: Moral ist wichtiger als Mammon, Deutsches Ärzteblatt Jg. 107, Heft 1-2, A7

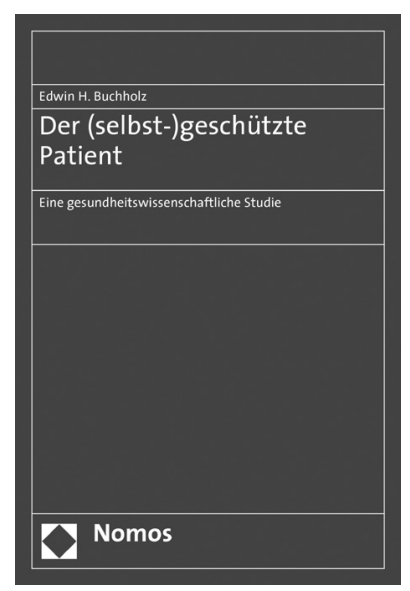

\section{Der (selbst-)geschützte Patient \\ Eine gesundheitswissenschaftliche Studie \\ Von Prof. Dr. Edwin H. Buchholz}

2010, 271 S., brosch., 39,-€, ISBN 978-3-8329-5236-5

Dieses Spätwerk des in Theorie und Praxis ausgewiesenen Autors ist eine Fundgrube für Fachleute und Laien. Im Zentrum steht die Schwachstelle Patient mit ihren Organisierungsproblemen und gravierenden Interessenverletzungen in der Behandlung. Die Stärkung des Patienten wird vor allem in größerer Eigenverantwortlichkeit gesehen.

Bitte bestellen Sie im Buchhandel oder versandkostenfrei unter $\boldsymbol{\sim}$ www.nomos-shop.de

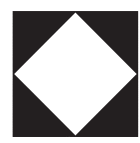

Nomos 